\title{
Commentary
}

\section{Changing the Irish dietary guidelines to incorporate the principles of the Mediterranean diet: proposing the MedÉire diet}

\author{
Audrey C Tierney ${ }^{1,2, *}$ and loannis Zabetakis $^{3}$ \\ ${ }^{1}$ School of Allied Health, HS2 032 Health Science Building, University of Limerick, Castletroy, Limerick, Republic of \\ Ireland: ${ }^{2}$ School of Allied Health, La Trobe University, Melbourne, Australia: ${ }^{3}$ Department of Biological Sciences, \\ University of Limerick, Limerick, Republic of Ireland
}

Submitted 15 February 2018: Final revision received 28 July 2018: Accepted 22 August 2018: First published online 15 0ctober 2018

\begin{abstract}
Objective: In Ireland, the major causes of death are CVD. The current Irish healthy eating guidelines and food pyramid primarily advocate a low-fat diet. However, there is overwhelming scientific evidence for the benefits of a Mediterranean diet (Med Diet) in the prevention and management of metabolic disease as well as improving overall health and well-being. In the current commentary, the rationale to incorporate the principles of the Med Diet into the Irish dietary guidelines is presented.

Design: Perspectives of authors.

Setting: Local and international.

Subjects: Populations in Europe, North America and Australia.

Results: Adopting components of the Med Diet presents a more evidence-based approach to updating the current Irish dietary guidelines. Experience and lessons from other non-Mediterranean countries show that it could be a feasible and effective solution to improving the dietary habits of the Irish population to prevent and mange chronic diseases.

Conclusions: Policies and programmes to address perceived barriers to the Med Diet's implementation and uptake in non-Mediterranean countries should be promoted.
\end{abstract}

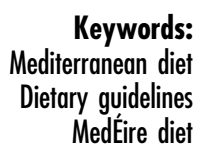

\section{Dietary guidelines in Ireland: current perspectives}

In the Republic of Ireland, it is currently estimated that $60 \%$ of adults and $25 \%$ of children are overweight or obese $^{(1,2)}$. In 2017, the total lifetime cost of childhood overweight and obesity alone was estimated at €4.6 billion $^{(3)}$.

We live in an obesogenic environment where work pattern changes, increased computerisation and improved infrastructure have changed the way we produce, process and distribute food, enabling easier access to unhealthful food choices ${ }^{(4)}$ driving obesity and metabolic diseases. Population physical activity levels have also decreased ${ }^{(5)}$ with technological advancements and reliance on motorised ways of transportation.

In Ireland, the leading cause of death is $\mathrm{CVD}^{(6)}$, with diet being a known modifiable risk factor. The purpose of the dietary guidelines in the Republic of Ireland is to encourage a healthy dietary pattern, to achieve optimal nutrition to maintain a healthy weight with the goal to promote a positive lifestyle for everyone and to prevent nutrition-related diseases such as CVD. The guidelines are depicted by a food pyramid display and while it has been found that the overall messages are well understood, the findings of the recent Healthy Ireland Survey 2015 demonstrate that they do not seem to be translated into daily food choices or population dietary pattern changes $^{(5)}$. In that survey, just $26 \%$ of the Irish population reported that they eat $\geq 5$ portions of fruit and vegetables daily and $65 \%$ reported that they consume snack foods or sugar-sweetened drinks daily ${ }^{(5)}$. Not only do the guidelines seem to be followed loosely, their evidence base and translational potential may need to be revisited in the light of the more recent evidence base presented in the current commentary. However, eating behaviours are complex in nature and a country's dietary guidelines do not solely determine consumption. Factors to be considered include an individual's preferences, lifestyle, culture, and the 
accessibility and affordability of food, which may impact upon the nutritional adequacy of the diet with other farreaching effects on the environment and economic landscapes $^{(7)}$.

\section{The Mediterranean diet: evidence of benefit for health and disease}

The best international evidence should be at the core and foundation of the development and redevelopment of all dietary guidelines. The Mediterranean diet (Med Diet) is one of the most globally researched and healthiest dietary patterns promoted for the management of chronic diseases and longevity ${ }^{(8,9)}$.

The traditional Med Diet is predominantly a plant-based diet, characterised by a high consumption of vegetables, fruits, nuts, legumes and unprocessed cereals; a low consumption of red meat, meat products and sweets; a moderate consumption of fermented dairy (cheese and yoghurt), poultry and fish; and with red wine consumed in moderation and with meals. As a percentage of energy, total fat content can be as high as $40 \%$ with over half being monounsaturated fat $(>20 \%)$. This is predominantly due to the liberal use of extra virgin olive oil (EVOO), the main source of added fat, which occupies a central position in the diet ${ }^{(10)}$.

While the Med Diet differs in its definition across the literature and regions, findings are consistent with respect to its many health benefits ${ }^{(11)}$. The beneficial health effects are owing primarily to the low intake of saturated fat and increased intakes of fibre, functional fatty acids and lipids, antioxidants and bioactive compounds.

The Med Diet is associated with reduced mortality ${ }^{(12,13)}$, reduction in the risk of $\mathrm{CVD}^{(14)}$ and incidence of $\mathrm{CHD}^{(15)}$, cancer ${ }^{(16-19)}$ and stroke ${ }^{(20)}$, and prevention of type 2 diabetes $^{(21,22)}$ and metabolic syndrome ${ }^{(23)}$. While the studies are largely observational, the landmark Spanish PREDIMED randomised controlled trial demonstrated a reduction in the risk of cardiovascular events by approximately $30 \%$ and a $52 \%$ reduction in diabetes incidence when participants followed a Med Diet supplemented with either nuts or EVOO ${ }^{(24,25)}$. Components of the Med Diet are also beneficial for weight loss in obesity and for maintaining a healthy weight in non-obese populations $^{(26,27)}$ and in type 2 diabetes mellitus ${ }^{(28)}$. The Med Diet has been shown to be beneficial in the treatment of depression ${ }^{(29)}$ and for global cognition ${ }^{(30-32)}$, with emerging evidence for benefits in disability and overall quality of life ${ }^{(33,34)}$. In a recent umbrella review of metaanalyses of observational studies and randomised controlled trials, the associations between adherence to a Med Diet and health outcomes for a population of over $12 \cdot 8$ million people were identified and affirmed ${ }^{(9)}$. A reduced risk of mortality, CVD, CHD, myocardial infarction, overall cancer incidence, neurodegenerative disease and diabetes with greater adherence to the Med Diet was found. Of note, the majority of these studies were carried out in Mediterranean countries; however, evidence is now emerging as to the beneficial effects of the Med Diet in non-Mediterranean areas.

Beyond its nutrient profile the Med Diet is palatable, sustainable and has low impact on the environment. This holistic diet provides opportunities for conserving food biodiversity and raising awareness of how to better use current agricultural systems, aiming to revive activities that minimise waste of natural resources in producing food for local and seasonal consumption ${ }^{(35)}$.

\section{Feasibility of translating the Mediterranean diet to an Irish population: lessons learned from overseas}

From epidemiological and interventional analysis, adoption of the Med Diet varied between 14.0 and $65.5 \%$ in Canada and North America(36-41). In a Swedish cohort, high adherence to a Med Diet was associated with lower cardiovascular mortality in women ${ }^{(42)}$, and in the EPIC (European Prospective Investigation into Cancer and Nutrition)-Norfolk study, adherence to a predefined Med Diet showed an inverse association with incident CVD and all-cause mortality $^{(43)}$. In Australia, a group of researchers employ a Cretan-based Med Diet protocol in dietary interventions in non-Med Diet populations to assess the effects on metabolic conditions ${ }^{(44-46)}$. Recently, this group has published strategies for translating the traditional Med Diet into non-Mediterranean settings, which include meal plans, practical strategies and examples to attain the key principles of the diet with foods that are available and accessible locally ${ }^{(47)}$. The Healthy Eating Pyramid in Australia $^{(48)}$ incorporates the principles of the Med Diet. Herbs and spices are included; legumes are in the 'eat most' section; a predominance of fruits and vegetables is supported; and olive oil has a place in the pyramid. The UK National Institute for Health and Care Excellence recommends a Mediterranean-style diet for the secondary prevention of $\mathrm{CVD}^{(49)}$. A UK population reported that the Med Diet allowed them to redefine cultural eating habits, introduced a better quality of food and reported several physical benefits ${ }^{(50)}$. However, barriers to its implementation in this and in a Northern Irish at-risk population included difficulty adapting to the eating pattern, limited knowledge of the diet, perception of expense and availability of foods, expectation of the time commitment to the diet, and resistance to dietary change and established eating habits ${ }^{(50,51)}$. The latter study serves well for policy makers to identify practical strategies to influence perceived barriers to bring about dietary behaviour changes towards a Med Diet in an Irish population.

While adopting a Med Diet in non-Mediterranean countries is a challenge, it is achievable. In 2016, the US National Heart, Lung, and Blood Institute agreed the importance of testing the translation of the Med Diet in the 
USA and proposed an alternative healthy Mediterraneanstyle eating pattern ${ }^{(52)}$, cognisant of local dietary practices and foods that do not have negative health effects and may be consumed alongside a Med Diet. For example, despite it not being an integral component of the Med Diet, the Dietary Guidelines for Americans 2015-2020 do not impose restrictions on egg consumption, because dietary cholesterol is not considered a concern ${ }^{(13,52)}$. This acknowledgement of incorporating aspects of local dietary practices may lead to better adoption and adherence to the new promoted guidelines and lead to significant changes to health. In addition, practical resources to promote the diet have been disseminated, aiming to easily apply the diet into the American setting. Authors have outlined practical recommendations as to how to shift current dietary practices in non-Mediterranean countries to more Med Diet pattern approaches, from replacing processed snacks with mixed tree nuts to substituting red or processed meat with seafood and legumes ${ }^{(14)}$.

\section{Proposing a MedÉire food pyramid}

Despite the substantial evidence in favour of a high-fat Med Diet, population and clinical guidelines continue to focus on low fat messages ${ }^{(53)}$. In the current Irish Food Pyramid ${ }^{(54)}$ increased consumption of fruits, vegetables and whole grains with small amounts of fats and oils are encouraged. The guidelines advise to replace high-fat foods that contain predominantly saturated fat (such as butter and cream) with foods that contain predominantly polyunsaturated and monounsaturated fats such as oils and spreads. Olive oil is grouped with other fats ('Choose rapeseed, olive, canola, sunflower or corn oils') and the message is to always cook with as little fat or oil as possible ${ }^{(54)}$, a message that is entirely opposite to the recommendations of the Med Diet pyramid. Seed oils are susceptible to oxidation when heated, due to the high polyunsaturated fat content and low levels of phenolics ${ }^{(55)}$. In contrast, EVOO is stable on prolonged heating, remaining rich in phenolic compounds and producing few potentially harmful polar compounds ${ }^{(56)}$. In recent years, evidence indicates that bioactive polyphenols present in EVOO contribute substantially to many health benefits ${ }^{(57)}$. The advice to consume 1 teaspoon of oil when cooking is in stark contrast and falls well below the recommended $50 \mathrm{ml}$ per person per day that has been associated with reduction in the risk of CVD as found in the landmark PREDIMED study ${ }^{(24)}$.

While we have positively moved away from a singlenutrient to a whole-of-diet approach with the Irish 2016 dietary guidelines and recommendations, there are still some inadequacies in the messages conveyed. It needs to be recognised that foods with similar fat contents can have very different nutritional values. Grouping together red meat with poultry and fish in the current Irish Food Pyramid, in the frequency promoted, is not reflective of the current evidence base and conveys to the public that these foods are of similar nutritional value; a message that is not entirely scientifically valid. Previous work has shown that fish ${ }^{(58)}$, olive oil ${ }^{(59,60)}$, fermented dairy products $^{(61-63)}$ and red wine $^{(64)}$ have strong anti-inflammatory activities protective against CVD. While red meat contains proteins of high biological value and important micronutrients such as $\mathrm{B}$ vitamins, iron (both free iron and haem iron) and zinc, in comparison to oily fish it has a higher saturated fat and lower $n$ - 3 fat content. Regular consumption of red meat has been associated with cancer risk ${ }^{(65)}$, heart disease ${ }^{(66)}$ and when cooked to a high temperature, type 2 diabetes risk ${ }^{(67)}$. Fish consumption, however, has been associated with a lower risk of CVD and CVD death ${ }^{(68)}$. For MedÉire, it is proposed to consume two or more servings of fish, less than two servings of red meat (preferably lean cuts) and less than one serving of processed meat every week. This frequency of consumption will provide the nutritional benefits that meat favours. This contrasts with the potential two servings of meat per day that the Irish population could consume according to the current recommended guidelines ${ }^{(54)}$.

The Med Diet with its basic themes is easily adaptable to an individual country's culture. In 2009 and 2010, through an international scientific consensus process, a new revised Med Diet pyramid was developed as a simplified main frame to be adapted to different countries' specific variations related to geographical, socio-economic and cultural contexts ${ }^{(69)}$, focusing on local, accessible and seasonal produce. Although not produced in Ireland, the staple component, EVOO, is readily accessible in all supermarkets, food stores and markets at a reasonable price, as are other key components such as canned or dry legumes and nuts. Other fundamental foods that constitute the Med Diet pattern - such as whole grains, leafy greens and all other vegetables, beans, fish, red and white meat, fresh fruit and dairy - are grown or produced in Ireland and are abundant and affordable, highlighting that the Med Diet can be adapted for the Irish context. A recent study examining the association between dietary cost and adherence to the Med Diet in a UK population found that adherence to the diet was associated with marginally higher dietary cost, but that the potential economic barriers of high adherence would be offset by cost saving from reducing unhealthy food consumption ${ }^{(70)}$. With many scientific and media reports increasingly being published on the health benefits of the Med Diet ${ }^{(8,9,71)}$, the key principles and ways of incorporating it into everyday life are becoming more culturally acceptable in nonMediterranean populations ${ }^{(36-38,43-51)}$.

The Med Diet frame also takes account of the concept of sustainability with the promotion of a predominantly plantbased dietary pattern, making use of natural resources and skills, conserving a fragile and strained ecosystem ${ }^{(69,72)}$. The base of the MedÉire food pyramid would be comprised of plant-origin foods; foods that sustain the diet and foods that the Irish agricultural and food systems could support ${ }^{(73)}$. 
Continued meat production is inefficient in terms of use of agricultural land, as more plant-based food is required for rearing of livestock ${ }^{(73)}$. The external costs of the current system in terms of climate change and food and nutrition security are increasing ${ }^{(73)}$. A redirection of land for the purposes of supporting growth of plant-based foods for human consumption is needed ${ }^{(74)}$.

The high nutritional quality of the Med Diet with its low environmental impact is a unique selling point of this diet. For the MedÉire diet, we propose the adaption of the current Irish Food Pyramid to incorporate its key principles. Consumption of vegetables, fish, olive oil and legumes in the MedÉire diet needs to be further encouraged. Given the findings of the Healthy Ireland Survey and recent studies in non-Mediterranean populations, reasons for suboptimal consumption of vegetables and barriers to adopting a Med Diet need to be explored. Information and education are key to implementation for it to become a feasible and sustainable diet for the Irish people and to overcome the main barriers including limited knowledge, expense, availability, and time and skill commitment ${ }^{(51)}$.

Addressing the education and knowledge components, incorporating the MedÉire diet into national policies and associated action plans around existing food- and dietrelated programmes is key to its uptake across all ages and stages.

The Healthy Eating and Active Living policy priority programme established in $2016^{(75)}$ aims to improve the health and well-being of service users, with a particular focus on families and children. Through the implementation of A Healthy Weight for Ireland: Obesity Policy and Action Plan 2016-2025(76), the number of people in Ireland eating a healthier diet by increased consumption of fruit and vegetables, reduced consumption of foods high in fat, salt and sugar, and achieving and maintaining a healthier weight, is encouraged. Activities as part of these programmes are ideal platforms for the promotion of a MedÉire diet. For example, healthy eating standards for school meals ${ }^{(77)}$ and the Schools Meals Programme ${ }^{(78)}$ are initiatives that can be easily targeted to teach and translate to young populations the staples of a Med Diet. Accessing main food staples such as olive oil, nuts, legumes, fish and fresh vegetables, and promoting the affordability of the Med Diet ${ }^{(79)}$ to quell misconceptions that the cost is significantly more, also need to be promoted.

\section{Conclusion}

Ireland is set to become one of the fattest nations in Europe. A drastic overhaul of diet and lifestyle practices is needed. Adopting components of the Med Diet may present a feasible and effective solution to improve our dietary habits and prevent and mange chronic diseases. The high-quality evidence base of the anti-inflammatory Med Diet pattern has not been observed for any other diet. Observational, interventional and meta-analyses support the consumption of a Med Diet for the prevention and reduction of CVD risk $^{(9,80)}$. In terms of an evidence base for promoting dietary patterns, the Med Diet has preventive and beneficial effects for CVD, diabetes, stroke and mental health - issues at the core of Irish society. Taking an approach like the USA, first testing the feasibility and acceptability of a Med Diet in the Irish population, is warranted. Examining trials that have been carried out in non-Mediterranean populations to devise the most appropriate dietary interventions and to address barriers to implementation and change is paramount. We suggest that adapting our food and dietary pattern guidelines towards a MedÉire-type dietary pyramid, following the principles of the Med Diet (i.e. using olive oil liberally; consuming vegetables at every meal; encouraging low meat intake (with some meat-free days), instead opting for oily fish and legumes), is of high importance and urgency. Following MedÉire, there is potential to change the overall macronutrient content of the diet by the addition of key staple and functional foods to protect against chronic diseases. In such an approach, we need to overcome barriers, commence promotion and education early, engage with government and industry stakeholders to make healthier food cheaper and more accessible, and market appropriately to inform the public of the benefits of the diet.

\section{Acknowledgements}

Financial support: This research received no specific grant from any funding agency in the public, commercial or notfor-profit sectors. Conflict of interest: The authors have no conflict of interest to declare. Authorship: A.C.T. and I.Z. devised the rationale and reasoning for the commentary. A.C.T. and I.Z. wrote the manuscript. Ethics of buman subject participation: Not applicable.

\section{References}

1. Irish Universities Nutrition Alliance (2011) National Adult Nutrition Survey Summary Report. https://irp-cdn.multiscreensite. com/46a7ad27/files/uploaded/The\%20National\%20Adult\% 20Nutrition\%20Survey\%20Summary\%20Report\%20March\% 202011.pdf (accessed September 2018)

2. Irish Universities Nutrition Alliance (2012) National Pre-School Survey: Summary Report. https://irp-cdn.multiscreensite.com/ 46a7ad27/files/uploaded/The\%20National\%20Pre-School\% 20Nutrition\%20Survey\%20Summary\%20Report\%20June\% 202012.pdf (accessed September 2018).

3. Perry IJ, Millar SR, Balanda KP et al. (2017) What are the estimated costs of childhood overweight and obesity on the island of Ireland? http://www.safefood.eu/SafeFood/media/ SafeFoodLibrary/Documents/Publications/Research\%20Reports/ Cost-of-childhood-obesity-Report.pdf (accessed September 2018).

4. Ley SH, Hamdy O, Mohan V et al. (2014) Prevention and management of type 2 diabetes: dietary components and nutritionals strategies. Lancet 383, 1999-2007. 
5. Ipsos MRBI (2015) Healthy Ireland Survey 2015: Summary of Findings. http://health.gov.ie/wp-content/uploads/2015/10/ Healthy-Ireland-Survey-2015-Summary-of-Findings.pdf (accessed September 2018).

6. Central Statistics Office (2016) CSO statistical publication, 31 May 2016, 11am. http://www.cso.ie/en/releasesandpublications/ ep/p-vsys/vitalstatisticsyearlysummary2015/ (accessed September 2018).

7. Brug J (2008) Determinants of healthy eating: motivation, abilities and environmental opportunities. Fam Pract 25, Suppl. 1, i50-i55.

8. Trichopoulou A, Martínez-González MA, Tong TYN et al. (2014) Definitions and potential health benefits of the Mediterranean diet: views from experts around the world. BMC Med 12, 112.

9. Dinu M, Pagliai G, Casini A et al. (2017) Mediterranean diet and multiple health outcomes: an umbrella review of metaanalyses of observational studies and randomised trials. Eur J Clin Nutr 72, 30-43.

10. Trichopoulou A, Costacou T, Mamja C et al. (2003) Adherence to a Mediterranean Diet and survival in a Greek population. N Engl J Med 348, 2599-2608.

11. Sofi F, Macchi C, Abbate R et al. (2014) Mediterranean diet and health status: an updated meta-analysis and a proposal for a literature-based adherence score. Public Health Nutr 17, 2769-2782.

12. Trichopoulou A, Orfanos P, Norat T et al. (2005) Modified Mediterranean diet and survival: EPIC-elderly prospective cohort study. BMJ 330, 991.

13. Trichopoulou A, Bamia C \& Trichopoulos D (2009) Anatomy of health effects of Mediterranean diet: Greek EPIC prospective cohort study. BMJ 338, b2337.

14. Martínez-González MA, Soledad Hershey M, Zazpe I et al. (2017) Transferability of the Mediterranean diet to nonMediterranean countries. What is and what is not the Mediterranean diet. Nutrients 9, E1226.

15. Mente A, de Koning L, Shannon HS et al. (2009) A systematic review of the evidence supporting a causal link between dietary factors and coronary heart disease. Arch Intern Med 169, 659-669.

16. Benetou V, Trichopoulou A, Orfanos P et al. (2008) Conformity to traditional Mediterranean diet and cancer incidence: the Greek EPIC cohort. Br J Cancer 99, 191-195.

17. Couto E, Boffetta P, Lagiou P et al. (2011) Mediterranean dietary pattern and cancer risk in the EPIC cohort. $\mathrm{Br} J$ Cancer 104, 1493-1499.

18. Trichopoulou A, Bamia C, Lagiou P et al. (2010) Conformity to traditional Mediterranean diet and breast cancer risk in the Greek EPIC (European Prospective Investigation into Cancer and Nutrition) cohort. Am J Clin Nutr 92, 620-625.

19. Bamia C, Lagiou P, Buckland G et al. (2013) Mediterranean diet and colorectal cancer risk: results from a European cohort. Eur J Epidemiol 28, 317-328.

20. Misirli G, Benetou V, Lagiou P et al. (2012) Relation of the traditional Mediterranean diet to cerebrovascular disease in a Mediterranean population. Am J Epidemiol 176, 1185-1192.

21. Martinez-Gonzalez MA, de la Fuente-Arrillaga C, NunezCordoba JM et al. (2008) Adherence to Mediterranean diet and risk of developing diabetes: prospective cohort study. BMJ 336, 1348-1351.

22. Salas-Salvado J, Bullo M, Estruch R et al. (2014) Prevention of diabetes with Mediterranean diets: a subgroup analysis of a randomized trial. Ann Intern Med 160, 1-10.

23. Kastorini CM, Milionis HJ, Esposito K et al. (2011) The effect of Mediterranean diet on metabolic syndrome and its components: a meta-analysis of 50 studies and 534,906 individuals. J Am Coll Cardiol 57, 1299-1313.

24. Estruch R, Ros E, Salas-Salvadó J et al.; PREDIMED Study Investigators (2013) Primary prevention of cardiovascular disease with a Mediterranean diet. N Engl J Med 368, 1279_ 1290.

25. Salas-Salvadó J, Bulló M, Babio N et al.; PREDIMED Study Investigators (2011) Reduction in the incidence of type 2 diabetes with the Mediterranean diet. Results of the PREDIMED-Reus nutrition intervention randomized trial. Diabetes Care 34, 14-19.

26. Shai I, Schwarzfuchs D, Henkin Y et al.; Dietary Intervention Randomized Controlled Trial (DIRECT) Group (2008) Weight loss with a low-carbohydrate, Mediterranean, or low-fat diet. $N$ Engl J Med 359, 229-241.

27. Mozaffarian D, Hao T, Rimm EB et al. (2011) Changes in diet and lifestyle and long-term weight gain in women and men. N Engl J Med 364, 2392-2404.

28. Ajala O, English P \& Pinkney J (2013) Systematic review and meta-analysis of different dietary approaches to the management of type 2 diabetes. Am J Clin Nutr 97, 505-516.

29. Jacka FN, O'Neill A, Opie R et al. (2017) A randomised controlled trial of dietary improvement for adults with major depression (the 'SMILES' trial). BMC Med 15, 23.

30. Loughrey DG, Lavecchia S, Brennan S et al. (2017) The impact of the Mediterranean diet on the cognitive functioning of healthy older adults: a systematic review and meta-analysis. Adv Nutr 8, 571-586.

31. Lourida I, Soni M, Thompson-Coon J et al. (2013) Mediterranean diet, cognitive function, and dementia: a systematic review. Epidemiology 24, 479-489.

32. Singh B, Parsaik AK, Mielke MM et al. (2014) Association of Mediterranean diet with mild cognitive impairment and Alzheimer's disease: a systematic review and meta-analysis. J Alzheimers Dis 39, 271-282.

33. Fransen HP, Beulens JW, May AM et al. (2015) Dietary patterns in relation to quality-adjusted life years in the EPICNL cohort. Prev Med 77, 119-124.

34. May AM, Struijk EA, Fransen HP et al. (2015) The impact of a healthy lifestyle on disability-adjusted life years: a prospective cohort study. BMC Med 13, 39.

35. Burlingame B and Demini S (2011) Sustainable diets: the Mediterranean diet as an example. Public Health Nutr 14, 2285-2287.

36. Abdullah MMH, Jones JPH \& Jones PJH (2015) Economic benefits of the Mediterranean-style diet consumption in Canada and the United States. Food Nutr Res 59, 27541.

37. Goulet J, Lamarche B, Nadeau G et al. (2003) Effect of a nutritional intervention promoting the Mediterranean food pattern on plasma lipids, lipoproteins and body weight in healthy French-Canadian women. Atherosclerosis 170, 11524 .

38. Gardener H, Wright CB, Gu Y et al. (2011) Mediterraneanstyle diet and risk of ischemic stroke, myocardial infarction, and vascular death: the Northern Manhattan Study. Am J Clin Nutr 94, 1458-1464.

39. Tangney CC, Kwasny MJ, Li H et al. (2011) Adherence to a Mediterranean-type dietary pattern and cognitive decline in a community population. Am J Clin Nutr 93, 601-607.

40. Leblanc V, Begin C, Hudon AM et al. (2014) Gender differences in the long-term effects of a nutritional intervention program promoting the Mediterranean diet: changes in dietary intakes, eating behaviors, anthropometric and metabolic variables. Nutr J 13, 107.

41. Zbeida M, Goldsmith R, Shimony $\mathrm{T}$ et al. (2014) Mediterranean diet and functional indicators among older adults in non-Mediterranean and Mediterranean countries. J Nutr Health Aging 18, 411-418.

42. Tognon G, Nilsson LML, Lissner L et al. (2012) The Mediterranean diet score and mortality are inversely associated in adults living in the subarctic region. $J$ Nutr 142, 15471553.

43. Tong TYN, Wareham NJ, Khaw KT et al. (2016) Prospective association of the Mediterranean diet with cardiovascular 
disease incidence and mortality and its population impact in a non-Mediterranean population: the EPIC-Norfolk study. BMC Med 14, 135.

44. Papamiltiadous ES, Roberts SK, Nicoll AJ et al. (2016) A randomised controlled trial of a Mediterranean Dietary Intervention for Adults with Non Alcoholic Fatty Liver Disease (MEDINA): study protocol. BMC Gastroenterol 16, 14 .

45. Ryan MC, Itsiopoulos C, Thodis T et al. (2013) The Mediterranean diet improves hepatic steatosis and insulin sensitivity in individuals with non-alcoholic fatty liver disease. $J$ Hepatol 59, 138-143.

46. Itsiopoulos C, Brazionis L, Kaimakamis M et al. (2011) Can the Mediterranean diet lower HbA1c in type 2 diabetes? Results from a randomized cross-over study. Nutr Metab Cardiovasc Dis 21, 740-747.

47. George ES, Kucianski T, Mayr HL et al. (2018) A Mediterranean model in Australia: strategies for translating the traditional Mediterranean Diet into a mulitcultural setting. Nutrients 10, E465.

48. Nutrition Australia (2013) Healthy Eating Pyramid. http:// www.nutritionaustralia.org/national/resource/healthy-eatingpyramid (accessed September 2018).

49. National Institute for Health and Care Excellence (2013) 1.2 Lifestyle changes after an MI. Myocardial infarction: cardiac rehabilitation and prevention of further MI, NICE Guidelines CG172. https://www.nice.org.uk/guidance/cg172/chapter/ 1-recommendations\#lifestyle-changes-after-an-mi2 (accessed September 2018).

50. Middleton G, Keegan R, Smith MF et al. (2015) Brief report: Implementing a Mediterranean diet intervention into a RCT: lessons learned from a non-Mediterranean based country. $J$ Nutr Health Aging 19, 1019-1022.

51. Moore SE, McEvoy CT, Prior L et al. (2017) Barriers to adopting a Mediterranean diet in Northern European adults at high risk of developing cardiovascular disease. J Hum Nutr Diet. Published online: 21 November 2017. doi: $10.1111 /$ jhn. 12523 .

52. US Department of Health and Human Services, National Heart, Lung, and Blood Institute (2016) The National Heart, Lung, and Blood Institute Workshop: 'Toward Testing the Effects of a Mediterranean Dietary Pattern on Cardiovascular and Other Diseases in the United States'. https://www.nhlbi. nih.gov/events/2016/national-heart-lung-and-blood-instituteworkshop-toward-testing-effects-mediterranean (accessed September 2018).

53. Stone NJ, Robinson J, Lichtenstein AH et al. (2014) 2013 ACC/AHA guideline on the treatment of blood cholesterol to reduce atherosclerotic cardiovascular risk in adults: a report of the American College of Cardiology/American Heart Association Task Force on Practice Guidelines. Circulation 129, 25 Suppl. 2, S1-S45.

54. Department of Health (2016) The Food Pyramid. https:// www.hse.ie/eng/about/who/healthwellbeing/our-priorityprogrammes/heal/heal-docs/food\%20pyramid\%20professional \%20version.pdf (accessed September 2018).

55. Choe E \& Min DB (2006) Mechanisms and factors for edible oil oxidation. Compr Rev Food Sci Food Saf 5,169-186.

56. De Alzaa F, Guillaume C \& Ravetti L (2018) Evaluation of chemical and physical changes in different commercial oils during heating. Acta Sci Nutr Health 2, 2-11.

57. Visiolo F \& Martinez-Gonzalez MA (2018) Olive oil and prevention of chronic diseases: summary of an international conference. Nutr Metab Cardiovasc Dis 28, 649-656.

58. Nasopoulou C, Karantonis HC, Perrea DN et al. (2010) In vivo anti-atherogenic properties of cultured gilthead sea bream (Sparus aurata) polar lipid extracts in hypercholesterolaemic rabbits. Food Chem 120, 831-836.
59. Karantonis HC, Antonopoulou S, Perrea DN et al. (2006) In vivo antiatherogenic properties of olive oil and its constituent lipid classes in hyperlipidemic rabbits. Nutr Metab Cardiovasc Dis 16, 174-185.

60. Tsantila N, Karantonis HC, Perrea DN et al. (2007) Antithrombotic and antiatherosclerotic properties of olive oil and olive pomace polar extracts in rabbits. Mediators Inflamm 2007, 36204.

61. Poutzalis S, Anastasiadou A, Nasopoulou C et al. (2016) Evaluation of the in vitro anti-atherogenic activities of goat milk and goat dairy products. Dairy Sci Technol 96, 317-327.

62. Lordan R, Tsoupras A \& Zabetakis I (2017) Phospholipids of animal and marine origin: structure, function, and antiinflammatory properties. Molecules 22, E1964.

63. Megalemou K, Sioriki E, Lordan R et al. (2017) Evaluation of sensory and in vitro anti-thrombotic properties of traditional Greek yogurts derived from different types of milk. Heliyon 3, e00227.

64. Xanthopoulou MN, Kalathara K, Melachroinou S et al. (2017) Wine consumption reduced postprandial platelet sensitivity against platelet activating factor in healthy men. Eur J Nutr 56, 1485-1492.

65. International Agency for Research on Cancer (2018) Red Meat and Processed Meat. IARC Monographs on the Evaluation of Carcinogenic Risks to Humans no. 114. Lyon: IARC.

66. Abete I, Romaguera D, Vieira AR et al. (2014) Association between total, processed, red and white meat consumption and all-cause, CVD and IHD mortality: a meta-analysis of cohort studies. Br J Nutr 112, 762-775.

67. Liu G, Zong G, Wu K et al. (2018) Meat cooking methods and risk of type 2 diabetes: results from three prospective cohort studies. Diabetes Care 41, 1049-1060.

68. Zheng J, Huang T, Yu Y et al. (2012) Fish consumption and CHD mortality: an updated meta-analysis of seventeen cohort studies. Public Health Nutr 15, 725-737.

69. Dernini S \& Berry EM (2015) Mediterranean diet: from a healthy diet to a sustainable dietary pattern. Front Nutr 2, 15 .

70. Tong TYN, Imamura F, Monsivais P et al. (2018) Dietary cost associated with adherence to the Mediterranean diet, and its variation by socio-economic factors in the UK Fenland study. Br J Nutr 119, 685-694.

71. BBC good food (2017) Why are Mediterranean diets so healthy? https://www.bbcgoodfood.com/howto/guide/ why-are-mediterranean-diets-so-healthy (accessed October 2018).

72. Bach-Faig A, Berry EM, Lairon D et al. (2011) Mediterranean diet pyramid today. Science and cultural updates. Public Health Nutr 14, 2274-2284.

73. An Taisce (2016) Ireland's Agricultural Sector's Role in Food Security in the Wake of Climate Change. http://www. antaisce.org/file/1593/download?token=BcZ28AuL （accessed October 2018).

74. Garnett T (2010) Livestock, feed and food security. Food Climate Research Network Briefing Paper. https://www. fcrn.org.uk/sites/default/files/FCRN_livestockfeed_foodsecurity. pdf (accessed October 2018).

75. Health Service Executive (2018) Healthy Eating and Active Living Programme. https:/www.hse.ie/eng/about/who/ healthwellbeing/our-priority-programmes/heal/ (accessed September 2018).

76. Department of Health (2015) A Healthy Weight for Ireland: Obesity Policy and Action Plan 2016-2025. https://health. gov.ie/wp-content/uploads/2016/09/A-Healthy-Weight-forIreland-Obesity-Policy-and-Action-Plan-2016-2025.pdf (accessed September 2018).

77. safefood (2015) The healthy incentive for pre-schools project. http://www.safefood.eu/Publications/Research-reports/ 
The-healthy-incentive-for-pre-schools-project.aspx (accessed September 2018).

78. Department of Employment Affairs and Social Protection (2018) Schools Meals Programme. http://www.welfare.ie/ en/Pages/School-Meals-Programme.aspx (accessed September 2018).
79. Drewnowski A \& Eichelsdoerfer P (2009) The Mediterranean diet: does it have to cost more? Public Health Nutr 12, 1621-1628.

80. Mente A, de Koning L, Shannon HS et al. (2009) A systematic review of the evidence supporting a causal link between dietary factors and coronary heart disease. Arch Intern Med 169, 659-669. 